Strahlenther Onkol 2014 · 190:777

DOI 10.1007/s00066-014-0704-3

Published online: 2 July 2014

c) Springer-Verlag Berlin Heidelberg 2014

Florian Würschmidt ${ }^{1} \cdot$ Solveigh Stoltenberg $^{2} \cdot$ Matthias Kretschmer $^{1}$. Cordula Petersen ${ }^{3}$

${ }^{1}$ Department of Radiotherapy \& Radiooncology, Radiologische Allianz, Hamburg, Germany

2 Department of Radiation Oncology, Klinikum rechts der Isar, TU München, Munich, Germany

${ }^{3}$ Center for Oncology, Department of Radiotherapy and Radio-Oncology, UKE, Hamburg, Germany

\title{
Erratum to: Incidental dose to coronary arteries is higher in prone than in supine whole breast irradiation
}

\section{Erratum to: Strahlenther Onkol 2014 \\ 190:563-568}

Unfortunately, erroneous author affiliations were published in the article "Incidental dose to coronary arteries is higher in prone than in supine whole breast irradiation."

The correct list of author affiliations is as follows:

Florian Würschmidt ${ }^{1}$, Solveigh Stoltenberg $^{2}$, Matthias Kretschmer ${ }^{1}$ and Cordula Petersen ${ }^{3}$

${ }^{1}$ Department of Radiotherapy and Radiooncology, Radiologische Allianz, Hamburg, Germany

${ }^{2}$ Department of Radiation Oncology, Klinikum rechts der Isar, TU München, Munich, Germany

${ }^{3}$ Center for Oncology, Department of Radiotherapy and Radio-Oncology, UKE, Hamburg, Germany

We apologize for any inconvenience caused.

\section{Corresponding address}

Prof. Dr. F. Würschmidt

Department of Radiotherapy \& Radiooncology

Radiologische Allianz

Mörkenstr. 47, 22767 Hamburg

Germany

wuerschmidt@radiologische-allianz.de
The online version of the original article can be found at doi:http://dx.doi.org/10.1007/s00066014-0606-4 